\title{
Applied \\ Functional Analysis \\ and \\ Partial Differential \\ Equations
}


This page is intentionally left blank 


\section{Applied \\ Functional Analysis and \\ Partial Differential Equations}

Milan Miklavčič

Michigan State University 


\section{Published by}

World Scientific Publishing Co. Pte. Ltd.

P O Box 128, Farrer Road, Singapore 912805

USA office: Suite 1B, 1060 Main Street, River Edge, NJ 07661

UK office: 57 Shelton Street, Covent Garden, London WC2H 9HE

\section{British Library Cataloguing-in-Publication Data \\ A catalogue record for this book is available from the British Library.}

First published 1998

Reprinted 2001

\section{APPLIED FUNCTIONAL ANALYSIS AND PARTIAL DIFFERENTIAL EQUATIONS}

Copyright $\odot 1998$ by World Scientific Publishing Co. Pte. Ltd.

All rights reserved. This book, or parts thereof, may not be reproduced in any form or by any means, electronic or mechanical, including photocopying, recording or any information storage and retrieval system now known or to be invented, without written permission from the Publisher.

For photocopying of material in this volume, please pay a copying fee through the Copyright Clearance Center, Inc., 222 Rosewood Drive, Danvers, MA 01923, USA. In this case permission to photocopy is not required from the publisher.

ISBN 981-02-3535-6 


\section{Contents}

\section{Preface}

1 Linear Operators in Banach Spaces 1

1.1 Metric Spaces . . . . . . . . . . . . . . . . . . . 1

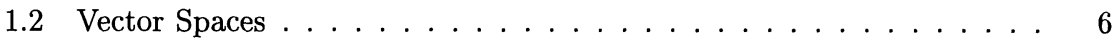

1.3 Banach Spaces $\ldots \ldots \ldots \ldots \ldots \ldots$

1.4 Linear Operators . . . . . . . . . . . . . . . . . . 11

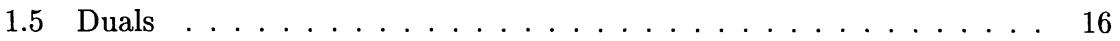

1.6 Spectrum . . . . . . . . . . . . . . . . . . 23

1.7 Compact Linear Operators $\ldots \ldots \ldots \ldots$

1.8 Boundary Value Problems for Linear ODEs . . . . . . . . . . . . 39

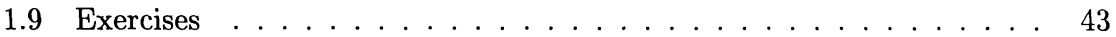

2 Linear Operators in Hilbert Spaces $\quad \mathbf{4 7}$

2.1 Orthonormal Sets . . . . . . . . . . . . . . . . . 47

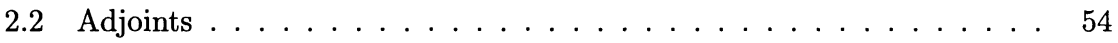

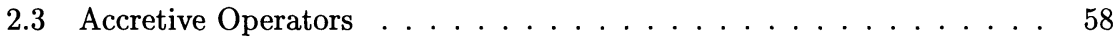

2.4 Weak Solutions . . . . . . . . . . . . . . . . . . 62

2.5 Example: Constant Coefficient PDEs . . . . . . . . . . . . . . . 68

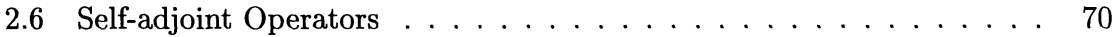

2.7 Example: Sturm-Liouville Problem . . . . . . . . . . . . . 76

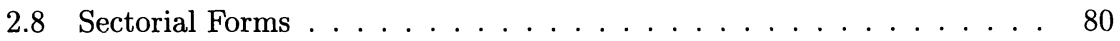

2.9 Example: Harmonic Oscillator and Hermite Functions $\ldots \ldots \ldots$

2.10 Example: Completeness of Bessel Functions . . . . . . . . . . . . 92

2.11 Example: Finite Element Method . . . . . . . . . . . . . . . 96

2.12 Friedrichs Extension $\ldots \ldots \ldots \ldots$

2.13 Exercises . . . . . . . . . . . . . . . . . . . . . 104

3 Sobolev Spaces 109

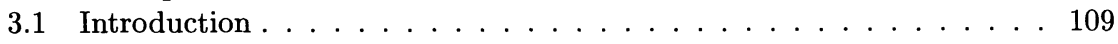

3.2 Fourier Transform . . . . . . . . . . . . . . . . . . . . 114

3.3 Distributions . . . . . . . . . . . . . . . . . . . 122 
3.4 Weak Derivatives . . . . . . . . . . . . . . . 126

3.5 Definition and Basic Properties of Sobolev Spaces . . . . . . . . . . . . 135

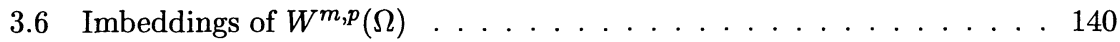

3.7 Elliptic Problems . . . . . . . . . . . . . . . . . . . . 153

3.8 Regularity of Weak Solutions . . . . . . . . . . . . . . . 160

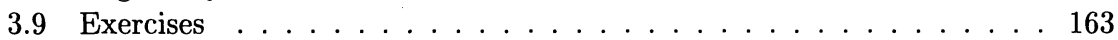

4 Semigroups of Linear Operators $\quad 167$

4.1 Introduction . . . . . . . . . . . . . . . . 167

4.2 Bochner Integral . . . . . . . . . . . . . . . . . . . . . . . . . . . . . . . . . . . .

4.3 Basic Properties of Semigroups . . . . . . . . . . . . . . . 176

4.4 Example: Wave Equation . . . . . . . . . . . . . . . . 187

4.5 Sectorial Operators and Analytic Semigroups . . . . . . . . . . . . 192

4.6 Invariant Subspaces . . . . . . . . . . . . . . . . . . . . 204

4.7 The Inhomogeneous Problem - Part I . . . . . . . . . . . . . . . 209

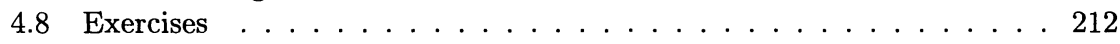

5 Weakly Nonlinear Evolution Equations 215

5.1 Introduction . . . . . . . . . . . . . . . . 215

5.2 Basic Theory . . . . . . . . . . . . . . . . . 218

5.3 Example: Nonlinear Heat Equation . . . . . . . . . . . . . . . . . 222

5.4 Approximation for Evolution Equations . . . . . . . . . . . . . . . 224

5.5 Example: Finite Difference Method . . . . . . . . . . . . . . 233

5.6 Example: Galerkin Method for Parabolic Equations . . . . . . . . . . 236

5.7 Example: Galerkin Method for the Wave Equation . . . . . . . . . . . 239

5.8 Friedrichs Extension and Galerkin Approximations . . . . . . . . . . . 243

5.9 Exercises . . . . . . . . . . . . . . . . 245

6 Semilinear Parabolic Equations $\quad \mathbf{2 4 7}$

6.1 Fractional Powers of Operators . . . . . . . . . . . . . . . . . 247

6.2 The Inhomogeneous Problem - Part II . . . . . . . . . . . . . . . . 254

6.3 Global Version . . . . . . . . . . . . . . . . . . . 257

6.4 Main Results . . . . . . . . . . . . . . . . . . . . 259

6.5 Example: Navier-Stokes Equations . . . . . . . . . . . . . . 267

6.6 Example: A Stability Problem . . . . . . . . . . . . . . . . 271

6.7 Example: A Classical Solution . . . . . . . . . . . . . . 273

6.8 Dynamical Systems . . . . . . . . . . . . . . . . . 276

6.9 Example: The Chafee-Infante Problem . . . . . . . . . . . . . . . 279

6.10 Exercises . . . . . . . . . . . . . . . . . 282 
This page is intentionally left blank 


\section{Preface}

This book is an introduction to partial differential equations (PDEs) and the relevant functional analysis tools which PDEs require. This material is intended for second year graduate students of mathematics and is based on a course taught at Michigan State University for a number of years. The purpose of the course, and of the book, is to give students a rapid and solid research-oriented foundation in areas of PDEs, like semilinear parabolic equations, that include studies of the stability of fluid flows and, more generally, of the dynamics generated by dissipative systems, numerical PDEs, elliptic and hyperbolic PDEs, and quantum mechanics. In other words, the book gives a complete introduction to and also covers significant portions of the material presented in such classics as Partial Differential Equations by Avner Friedman, Geometric Theory of Semilinear Parabolic Equations by Dan Henry, and Semigroups of Linear Operators and Applications to PDEs by A. Pazy.

The need for such a book is due to the fact that in order to study PDEs one needs to know some functional analysis, which requires a thorough knowledge of real analysis (Lebesgue integral). Therefore, if real analysis is studied in the first year of graduate school, and functional analysis in the second year, the student only begins with PDEs in the third year - and may even have to re-learn functional analysis if the prior instructor ignored unbounded operators (which sometimes happens).

The reader is expected to be comfortable with the Lebesgue integral; more specifically, with the material presented in Examples 1.3.4 and 1.5.2. The Cauchy Theorem is also used in a couple of places, with the most difficult version used in (4.44). These are the only real prerequisites for the whole book. Above this level, all theorems used are proved in the text. One may, and perhaps should, skip over some of the proofs. However, they are included in case they are needed.

With regard to the writing style, all formal statements, like Theorems, contain all assumptions except for those declared at the beginning of the section in which the statement appears. This should make it easy, even for a casual reader, to figure out what is actually assumed in a given statement. There is, however, one exception. Throughout Chapter 3 it is assumed, unless otherwise specified, that $\Omega$ is an arbitrary nonempty open set in $\mathbb{R}^{n}, n \in \mathbb{N}$.

In the first two chapters functional analysis tools are developed and differential operators are studied as examples. Sturm-Liouville operators are nice examples of self- 
adjoint operators with compact resolvent and are reused in Chapter 4 as generators of strongly continuous semigroups. Hörmander's treatment of weak solutions of constant coefficient PDEs is also presented early on as an example. The foundation of elliptic, parabolic and wave equations, as well as of Galerkin approximations, is given in the section on Sectorial Forms. Throughout the text, completeness of a number of orthonormal systems is proven.

The Fourier transform and its applications to constant coefficient PDEs are presented in Chapter 3. We briefly touch upon distributions and fundamental solutions, and prove the Malgrange-Ehrenpreis Theorem. Most of Chapter 3 is devoted to study of Sobolev spaces. Many sharp results concerning existence and compactness of imbeddings, as well as interpolation inequalities, are proven. These results are applied to elliptic problems in the last two sections.

The study of evolution equations begins in Chapter 4 where the semigroup theory is introduced. The Hille-Yosida Theorem for strongly continuous semigroups and Hille's construction of analytic semigroups are presented. The semigroup theory and the results of the previous chapters enable us to discuss linear parabolic and wave equations. In preparation for studies of nonlinear evolution equations, the invariant subspaces associated with the semigroups and the inhomogeneous problem are also examined.

A dynamical systems approach to weakly nonlinear evolution equations is given in Chapter 5 with a nonlinear heat equation studied as an example. Trotter's approximation theory is adapted to such equations giving convergence of Galerkin and finite difference type approximations.

The chapter on semilinear parabolic equations begins with a very technical section on fractional powers of operators. Our main results contain existence, uniqueness, continuous dependence, maximal interval of existence, stability and instability results. These results are applied to the Navier-Stokes equations, to a stability problem in fluid mechanics, to showing how a classical solution can be obtained, and to the ChafeeInfante problem as an example of a gradient system.

I wish to thank S. N. Chow and D. R. Dunninger for their early encouragement and my wife Pam for checking the grammar. 